Vol.(7)Issue(4), pp.330-343

DOI: http://dx.doi.org/10.21172/1.74.046

e-ISSN:2278-621X

\title{
DESIGN AND IMPLEMENTATION OF AN ENGINE CONTROL STRATEGY DURING GEAR SHIFTING PROCESS OF AUTOMATED MANUAL TRANSMISSION (AMT) FOR IMPROVEMENT OF SHIFT QUALITY AND FUEL EFFICIENCY
}

Anjus George ${ }^{1}$

\begin{abstract}
Automated Manual Transmission (AMT) combines comfort of use with a reduction in energy consumption, with production costs that are consequently lower compared to traditional automatic transmissions. AMT is based on an electronic control unit and a mechanical system that supervise the use of the clutch and gear shifting, allowing the driver to change gear without using the clutch, either sequentially or fully automatically. This project aims to improve the shift quality and reduce fuel consumption by means of control of engine speed and torque during shift process. Before shifting to neutral gear the engine torque is reduced to a minimum level. The position of the shift motor is controlled effectively for shift off and shift on process. Before changing to new gear position the engine speed is controlled as per shift request i.e., upshift or downshift, after that gear engagement is performed and the engine torque is restored as per driver's request. A virtual torsion sensor is designed using Kalman filter for estimating the torque transmitted through the drive shaft and a PID controller is designed to control this torque to a minimum level. The position of the gear actuator is also driven to reference position using PID mechanism. For speed control the advantage of both time-optimal control such as bang bang control and PID control are utilized.
\end{abstract}

\section{INTRODUCTION}

The engine of Automated Manual Transmission (AMT) system is a highly nonlinear, large inertia, parameter time varying, easily disturbed complex system. The time taken for the gear shift process is very short. Therefore, the control of the engine parameters such as speed and torque is complex and requires high performance.

The gear shift is achieved by replacing the automatic clutch by engine control. The conventional shifting process can be divided into seven operating phases: gear engaged, clutch release, gear shift off, gear selection, gear shift on, clutch engagement and engine torque restoration. The shifting process without the use of the clutch can be divided into five phases: engine torque reduction, gear shifting off, engine speed regulation, gear shifting on and engine

${ }^{1}$ Department of of Electronics Engineering Govt. Model Engineering College, Kochi 
torque restoration [1]. First the system undergoes a torque control phase in which the engine is controlled to a torque level corresponding to zero transferred torque in the transmission. Next in the speed synchronization phase, the gear disengages completely, and the gear selection starts as soon as the shifting off action is completed. The engine speed regulation is carried out simultaneously. Subsequently new gear is engaged and the engine torque is restored back to the level that the driver has commanded by the gas pedal.

In the torque control phase the drive shaft torque is estimated using a virtual torsion sensor and is controlled using a PID controller. To accomplish the process of gear shifting, the position of the actuator must be controlled precisely. To account for the performance and the dynamic behavior of the actuator motor, this paper implements a PID controller. The engine speed control is divided into downshift and up-shift process. Considering the advantages of both time-optimal control and PID control, a combined control strategy for engine speed during the gear-shifting process is proposed. The controller chooses between time optimal control and bang bang control through a mode switching mechanism.

\section{LITERATURE REVIEW}

The engine parameters like speed and torque are controlled during the gear shift process to improve shift quality and to reduce fuel consumption. Different methods are proposed for the optimal control of gear shift in automated manual transmissions.

Luigi Gilelmo et. al. in Ref [7] proposed a gear shift control strategy in which a controller is designed through a hierarchical approach by discriminating among five different AMT operating phases: engaged, slipping-opening, synchronization, go-to slipping, and slipping closing. The control schemes consist of decoupled and cascaded feedback loops based on measurements of engine speed, clutch speed, and throwout bearing position, and on estimation of the transmitted torque.

Magnus Petterson et. al. in Ref [2] put forward a method of gear shifting by engine control. In this the emphasis is given to minimize the total time needed for a gear shift, but when doing so driveline resonances may be excited. This in turn may lead to problems with disengaging the old gear and synchronizing speeds for engaging the new gear. Internal driveline torque control is a novel idea for handling resonances and increasing shift quality. By estimating the transmitted torque and controlling it to zero by engine control, the gear can systematically be disengaged with minimized driver disturbances and faster speed synchronization. In this a PID controller is designed to control the drive shaft torsion and to damp the driveline resonances.

Viet Ngo et. al. in Ref [9] suggested a design method for energy management strategy to explore the potential fuel saving of a hybrid electric vehicle (HEV) equipped with an automated manual transmission. The control algorithm is developed based on the combination of dynamic programming (DP) and Pontryagin's minimum principle (PMP) to optimally control the discrete gearshift command, in addition to the continuous power split between the internal combustion engine and the electric machine.

Zaimin Zhong et. al. in Ref [3] put forward that in automated mechanical transmission the shift quality can be significantly improved by means of the control of the engine speed. The engine speed control is be divided into upshift control and downshift control. During up-shift, the Bang-Bang algorithm and PID algorithm are integrated. During downshift, the PID control algorithm alone is used.

G Kong et. al. in Ref [1] proposed a control algorithm that combines speed and torque control of AMT vehicle powertrain to achieve shifting control without using the clutch. The torque control is achieved using PID mechanism. To realize accurate engine speed control, a combined control algorithm based on feed-forward, bangbang and PID control is adopted. Additionally, an optimized closed-loop position control algorithm based on LQR is proposed for the shift actuators.

\section{MOTIVATION AND OBJECTIVE}

The objective of the AMT is to automate the clutch and gearbox to increase driver comfort. With this approach the clutch is replaced by engine control. It is also necessary to control the engine speed and torque during shift process to reduce fuel consumption, engine noise, shift jerk, and clutch friction work. By the implementation of automated clutch, the service lives of the clutch components are lengthened, while fuel consumption and the associated CO2 emissions can be reduced.

It is crucial to control engine parameters during the gear shifting process of AMT for the purpose of reducing fuel consumption, engine noise, shift jerk and clutch friction work. The main objectives of the project are, 
1. To design a virtual drive shaft torsion sensor and a PID controller respectively, to estimate the drive shaft torque and to reduce it to a minimum level.

2. To design a position error closed loop controller by using PID controller, for controlling the position of a shift actuator precisely.

3. To design bang bang and PID controllers to control the engine speed during gear shift process.

4. Implement the complete strategy in LPC1768 platform.

\section{SOME BASIC CONCEPTS}

\section{- Vehicle Drive Train of AMT}

In recent years Automated Manual Transmission (AMT) attracts the driver's attention. It is mostly the same as manual transmission except for some extra features. Compared with manual transmission, the major difference is that the clutch actuator and synchronizers used in a gear box for a gear shift are servo-actuated hydraulically or electro-mechanically. An AMT is composed of a dry clutch, a gearbox and an embedded dedicated control system that uses electronic sensors, processors and actuators to actuate gearshifts on the driver's command. This removes the need for a clutch pedal while the driver still able to decide when to change the gear. The clutch itself is actuated by electronic equipment that can synchronize the timing and the torque required to make gear shifts quick and smooth. The system is designed to provide a better driving experience, especially in cities where congestion frequently causes stop-and-go traffic patterns.

The structural diagram of automotive power-train is shown in Fig 1 which is mainly composed of engine, clutch, wheel, main reducer and transmission which consist of five forward gears and one reverse gear.

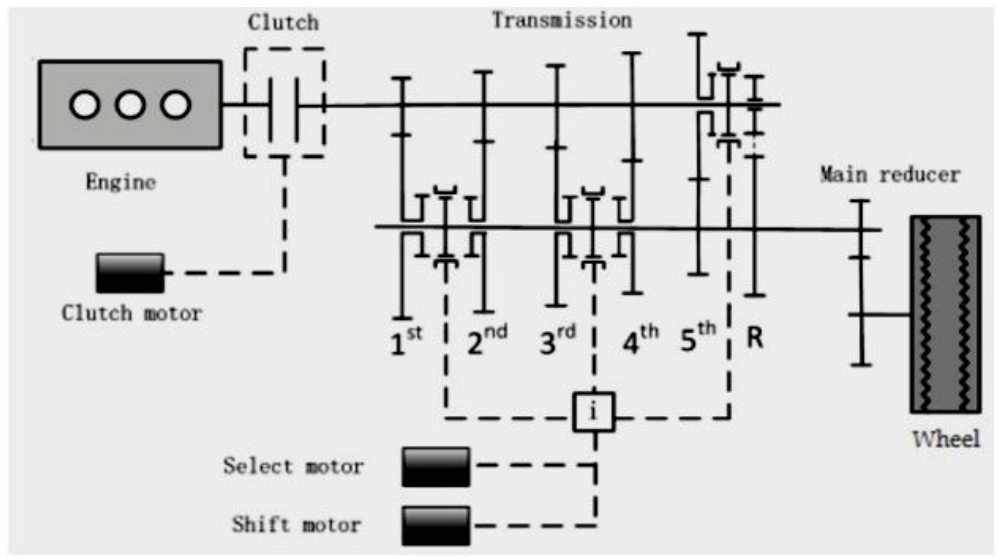

Figure 1. Structural diagram of vehicle drive-train with AMT.[1]

The process of shifting without using the clutch can be divided into five phases: decreasing the engine torque, engagement of neutral gear, speed synchronization, engagement of new gear and restoring the engine torque.

First, control is transferred from the driver to the control unit, entering the torque control phase. The engine is controlled to a torque level corresponding to zero transferred torque in the transmission. After neutral gear is engaged, the speed synchronization phase is entered. Then the engine speed is controlled to track the transmission speed (scaled with the conversion ratio of the new gear), where after the new gear is engaged.

Finally, the torque level is transferred back to the level that the driver demands. It is important to minimize the total time needed for a gear shift, since the vehicle is free-rolling with zero transmitted torque in the transmission. Furthermore, it is important to minimize the wear of the transmission, the noise, and the speed impulses[2].

\section{- Drive Shaft Flexibility}

The most critical and important part of the shift sequence is the torque control phase. Large torque change means that driveline resonances are excited. The main flexibility in the driveline is the drive shaft [2]. The drive shaft connects the transmission to the wheels. A simplified model of AMT drive train is shown in Fig 2. When the drive shaft is oscillating, the transmitted torque in the transmission also oscillates.

Since no sensor is there to measure the torque transmitted in the transmission, other indications like amplitude of the oscillations in transmission speed, time delay from commanded to accomplished disengaged gear etc must be used. 
Low amplitude of oscillations indicate less time delay and hence small transmitted torque. In Ref [5] it is proved that the amplitude of the oscillations in transmission speed increases due to increased drive shaft torsion. Therefore to reduce oscillations (to minimize transmission torque) it is sufficient to control drive shaft torsion to zero.

Shifting to neutral gear in a torque free state is very much difficult. In order to minimize the time needed for the torque control phase, the oscillations must be damped, which can be done by controlling the engine in an appropriate way. The torque control phase is even trickier if the driveline is oscillating prior to the gear shift, which sometimes happens with towed trailers or road roughness. If a gear is disengaged at a torque level different from zero, a speed impulse will follow which increases the wear of the transmission and disturbs the driver.

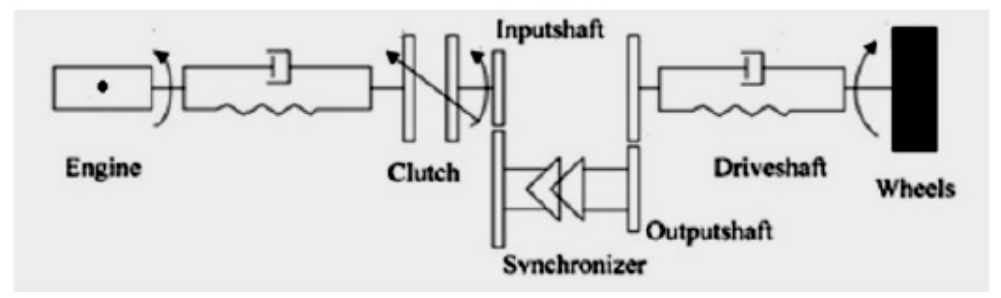

Figure 2: Simplified model of AMT drive train.[3]

- Drive Shaft Model

Magnus Petterson in Ref [5] proposed one simple model with drive shaft flexibility. The drive shaft can be modeled as a damped torsional flexibility which leads to the driveline model shown in Fig 3.

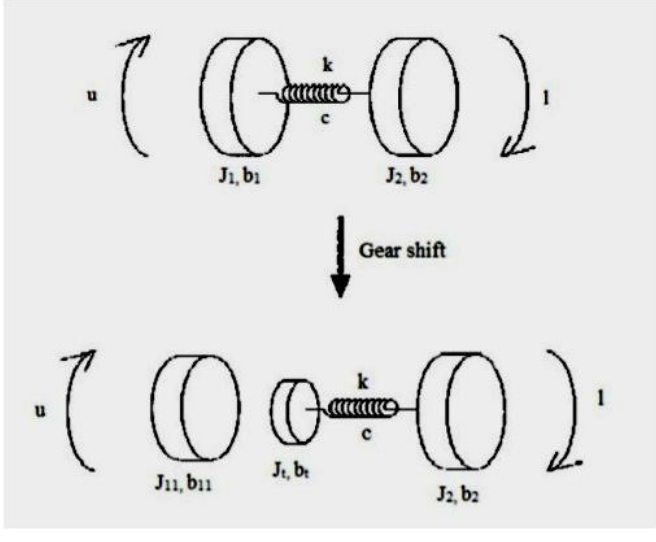

Figure 3: Driveline model before and after gear shift.[5]

In Fig 3, the engine, the clutch, the transmission, and the propeller shaft are lumped together as one rotating inertia $\mathrm{J} 1$, and the drive shaft connects this inertia to the inertia of the wheel $\mathbf{J} 2$. The wheel inertia is affected by the load l, which consists of the air drag, rolling resistance, and vehicle mass. In Fig 3, $\mathrm{u}$ is the throttle input and $\mathrm{k}$ and $\mathrm{c}$ are torsional stiffness and torsional damping of the drive shaft respectively. Viscous friction is assumed for both the rotating inertia with labels b1 and b2. Fig 3 also describes how the driveline model changes after neutral gear is engaged. The main flexibility is still the drive shaft, but the first inertia J1, is separated into two parts. The left model is the engine and left part of the transmission (characterized by J11, b11). The right model is a similar springmass model as before the gear shift, but with a significantly smaller first inertia (characterized by Jt, bt). The model prior to the gear shift is called the lumped drive line model, and the model after disengaged gear is called the decoupled model.

- State Space Model

Based on the above model (Fig 3) a state space model is derived which is used in the design of virtual torsion sensor. The state space representation of this model is,

$$
\dot{x}=\mathrm{Ax}+\mathrm{Bu}+\mathrm{Hl}
$$

where $\mathrm{A}, \mathrm{B}, \mathrm{H}, \mathrm{x}$ and $\mathrm{l}$ are defined as,

$$
\begin{aligned}
& x_{1}=\theta_{m} / i_{t} i_{f}-\theta_{w} \\
& x_{2}=\dot{\theta}_{m}
\end{aligned}
$$




$$
\begin{aligned}
& x_{3}=\dot{\theta}_{w} \\
& l=r_{w} m\left(c_{r 1}+g \sin (\alpha)\right)+\frac{1}{2} c_{w} A_{a} \rho_{a} r_{w}^{2} \dot{\theta}_{w}^{2}
\end{aligned}
$$

giving,

$$
\begin{gathered}
A=\left[\begin{array}{ccc}
0 & 1 / i & -1 \\
-k / i J_{1} & -\left(b_{1}+c / i^{2}\right) / J_{1} & c / i J_{1} \\
k / J_{2} & c / i J_{2} & -\left(c+b_{2}\right) / J_{2}
\end{array}\right] \\
B=\left[\begin{array}{c}
0 \\
1 / J_{1} \\
0
\end{array}\right] \\
H=\left[\begin{array}{c}
0 \\
0 \\
-1 / J_{2}
\end{array}\right]
\end{gathered}
$$

where the parameters are defined as,

$$
\begin{aligned}
& i=i_{t} i_{f} \\
& J_{1}=J_{m}+J_{t} / i_{t}^{2}+J_{f} / i_{t}^{2} i_{f}^{2} \\
& J_{2}=J_{w}+m r_{w}^{2} \\
& b_{1}=b_{t} / i_{t}^{2}+b_{f} / i_{t}^{2} i_{f}^{2} \\
& b_{2}=b_{w}+m c_{r 2} r_{w}^{2}
\end{aligned}
$$

where,

$\theta_{m}=$ Rotational displacement of engine crankshaft $(\mathrm{rad})$

$\theta_{w}=$ Rotational displacement of wheel ( $\mathrm{rad}$ )

$\dot{\theta}_{m}=$ Angular velocity of engine $(\mathrm{rad} / \mathrm{s})$

$\dot{\theta}_{w}=$ Angular velocity of wheel $(\mathrm{rad} / \mathrm{s})$

$r_{w}=$ Radius of the Tyre $(m)$

$m=$ Mass of vehicle $(\mathrm{kg})$

$c_{r 1}, c_{r 2}=$ Rolling resistance coefficients

$g=$ Acceleration due to gravity $\left(\mathrm{m} / \mathrm{s}^{2}\right)$

$c_{w}=$ Air drag coefficient

$A_{a}=$ Vehicle cross section area $\left(m^{2}\right)$

$\alpha=$ Angle between horizontal surface and slope plane (road slope) (rad)

$\rho_{a}=$ Air density $\left(\mathrm{kgm}^{3}\right)$ 


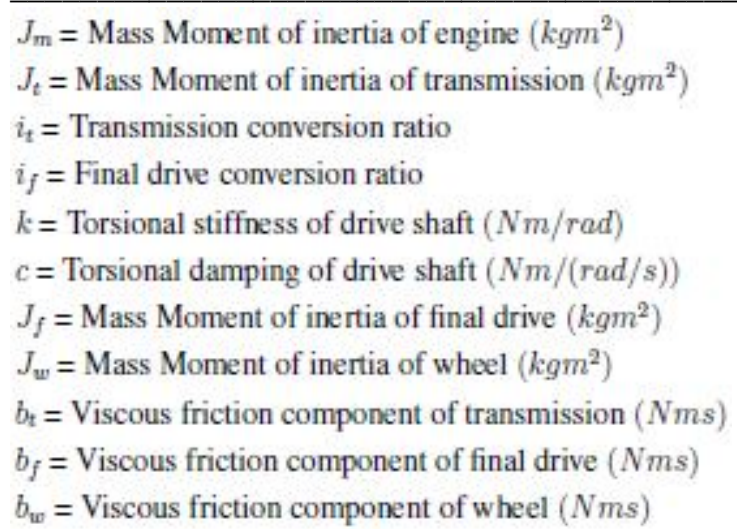

This model is described by three states which are drive shaft torsion (x1), engine speed (x2) and wheel speed (x3). The output torque of the engine is the driving torque $(\mathrm{Mm})$ subtracted by the engine friction (Mfr). The signal, $\mathrm{u}=\mathrm{Mm}-\mathrm{Mfr}$ is treated as control signal, since Mm can be controlled by injecting different amounts of fuel in the cylinders. The engine friction Mfr is function of engine speed and engine temperature [2]. The total torque transmitted in the drive shaft is given by $\mathrm{kx} 1+\mathrm{c}(\mathrm{x} 2 / \mathrm{i}-\square \mathrm{x} 3)$.

In AMT for gear change, the position of the shaft of these motors must be accurately controlled. In order to design such position control systems it is necessary to obtain, a mathematical model for the motor or system to be controlled. The main control mechanisms used in this paper are PID control and Bang bang control.

The PID controller is the most common form of feedback. The PID algorithm is described by:

$$
u(t)=K\left(e(t)+1 / T_{i} \int e(\tau) d(\tau)+T_{d} \frac{d e(t)}{d(t)}\right)
$$

where e is the error signal that is difference between the measured process variable and the reference variable. The control signal is thus a sum of three terms: the P-term which is proportional to the error e, the I-term which is proportional to the integral of the error e, and the $\mathrm{D}$-term which is proportional to the derivative of the error e. The controller parameters are proportional gain $\mathrm{K}$, integral time $\mathrm{Ti}$, and derivative time $\mathrm{Td}$.

In control theory, a bang-bang controller (on-off controller), also known as a hysteresis controller, is a feedback controller that switches abruptly between two states. In optimal control problems, it is sometimes the case that a control is restricted to be between a lower and an upper bound. If the optimal control switches from one extreme to the other at certain times then that control is referred to as a bang-bang solution.

A Kalman filter is an optimal estimator, i.e., infers parameters of interest from indirect, inaccurate and uncertain observations. It is recursive so that new measurements can be processed as they arrive. If all noise is Gaussian, the Kalman filter minimizes the mean square error of the estimated parameters. Given only the mean and standard deviation of noise, the Kalman filter is the best linear estimator. Non-linear estimators may be better. The process of finding the best estimate from noisy data amounts to filtering out the noise. However a Kalman filter also doesn't just clean up the data measurements, but also projects these measurements onto the state estimate.

\section{BLOCK DIAGRAM AND CONTROL STRATEGY}

The block diagram of proposed strategy is shown in Fig 4 .

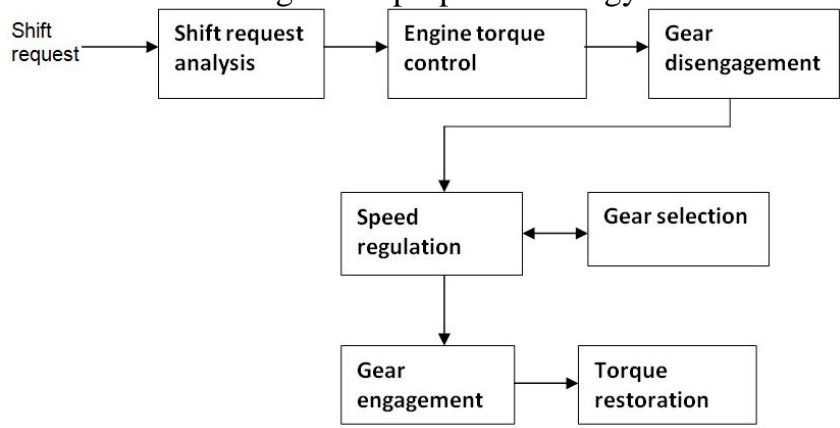

Figure 4: Block diagram of the proposed strategy

The clutch and the synchronizer stay engaged before shifting. Thus, the powertrain can be considered a closed system, and the clutch rotational speed is proportional to the transmission output shaft speed. The main 
characteristic of shift off phase is that the clutch is kept closed from shifting off until the gears engage again. Thus, the inertia of the driving components of the synchronizer includes the inertias of the engine, the clutch, the transmission input shaft and the constant-mesh gears. The engine speed regulation should be started soon after shifting off, and gear selection can be performed in parallel with the speed regulation. Generally, the speed regulation process takes longer than the gear selection, and thus the shifting on action can proceed after the speed regulation is accomplished.

Shifting off can be easily achieved without excessive wear of the gear faces when zero torque is transmitted to the driving shaft of the synchronizer. It is easy to shift off in the conventional shifting process because the clutch is released, and no torque exists on the driving shaft of the synchronizer. Thus, the engine torque should be decreased as much as possible, when shifting off without using the clutch such that the engine output torque is sufficient only to overcome its internal resistance and no torque is transferred to the driving shaft of the synchronizer.

In the speed regulation phase, the gear disengages completely, and the gear selection starts as soon as the shifting off action is completed. The engine speed regulation is carried out simultaneously. The goal of this phase is to adjust the engine speed as quickly as possible to the ideal velocity required by each gear ratio. The connection between the engine and the wheel is assumed to be rigid.

The goal of the torque restoration phase is to complete the gear shifting on process as quickly as possible and subsequently to restore the engine torque to the level that the driver has commanded by the gas pedal. The shift on action can be performed soon after the engine speed reaches the target engine speed. The engine torque should be restored to the commanded level at an appropriate rate to avoid affecting the quality of the shift.

In shift request analysis two blocks are used. Up/down finder and speed finder. The up/down finder is implemented as a comparator which compares the current and target gear no. and finds whether the request is for upshift or downshift. The speed finder block has inputs current vehicle speed, current gear ratio, target gear ratio, final drive ratio and radius of tire and the outputs are current engine speed, target engine speed and the speed difference between the two.

In Ref [5] it was shown that the oscillations in the transmission speed could be explained by a nonzero drive-shaft torsion. Hence the aim is to control the drive shaft torsion. The estimation of the drive-shaft torsion is more easily performed than estimating the transmission torque, since the different behavior for each gear can be neglected, as the drive shaft is the same for all gears. Thus the torque control phase consist of two sub phases: estimation of torque and control of the same. It consists of two blocks, a virtual torsion sensor and a PID controller. The virtual torsion sensor is used to estimate the drive shaft torsion and is implemented using kalman filter. The inputs to the sensor are engine speed or wheel speed. The output of the sensor is the estimated drive shaft torsion. The PID controller will make the estimated torque to zero.

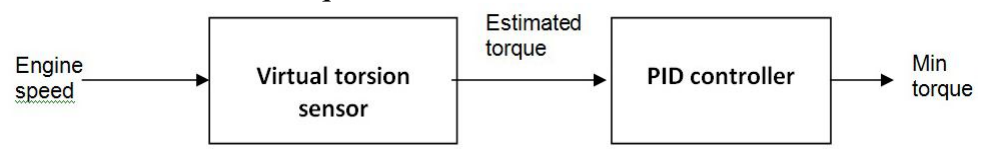

Figure 5: Torque control strategy.

The position of the motor actuator is controlled in the gear disengagement, selection and engagement phase. The gear is selected according to the target gear position. Then the dc motor is actuated to that selected gear position. For position control a PID controller is used. The current gear position and target gear position are compared and the error is minimized using controller.

The speed regulation phase is implemented to control the engine speed during gear shift. If upshift is initiated then engine speed should be reduced. Therefore a combined algorithm using bang bang and PID control is implemented during up-shift. During downshift the engine speed should be increased. Since this is comparatively easier than upshift only PID controller is implemented during downshift. In both cases the error between current speed and target speed are calculated and controlled. During up-shift if the error is greater than a threshold value bang bang controller action takes place. Otherwise mode is switched to PID controller. The bang bang controller controls the torque whereas, the PID controller controls the speed.

After the new gear is engaged the torque is restored as per driver's request. Each gear has a different torque requirement. This torque is called stable torque. The stable torque is predetermined and saved. Next this torque is given as the the input to the actuator.

The driver torque map is plotted as a function of engine speed and accelerator pedal position. The engine torque map is plotted as torque delivered by engine as a function of engine speed and engine throttle position. The engine torque map is used to position the engine throttle to match the driver's torque demand.

\section{SYSTEM DESIGN AND IMPLEMENTATION}


The proposed model consists of four forward gears. Therefore the current and target gear number can be from one to four. The up-shift considered for the design process is sequential. The speed finder will check the current and target gear combinations and using corresponding vehicle speed and gear ratios it will calculate target engine speed and current engine speed. The formulas used to calculate current engine speed, speed difference target engine speed are given below.

$$
\begin{aligned}
& \Delta n=\frac{\left(i_{g_{k}+1}-i_{g k}\right) * i_{f} * v(k)}{0.377 * r_{w}} \\
& w_{e}(k)=\frac{v(k) * i_{g_{k}} * i_{f}}{0.377 * r_{w}} \\
& w_{e}(k+1)=w_{\varepsilon}(k)+\Delta n
\end{aligned}
$$

where,

$\Delta n=$ Speed difference $(r p m)$

$i_{g_{k+1}}=$ Target gear ratio

$i_{g_{k}}=$ Current gear ratio

$v(k)=$ Vehicle speed $(k m p h)$

$w_{k}(k)=$ Current engine speed $(r p m)$

$w_{\varepsilon}(k+1)=$ Target engine speed $(r p m)$

The virtual drive shaft torsion sensor is implemented using Kalman filter. The linearized model with disturbances is given by,

$\dot{x}=A \Delta x+B \Delta u+G w$

and

$y=C \Delta x+v$

where the matrices A, B, G, C are given by,

$$
\begin{gathered}
A=\left[\begin{array}{ccc}
-0.2348 & -3.805 & -3.131 \\
-0.05674 & 4.584 & 4.476 \\
1.781 & -6.631 & -5.49
\end{array}\right] \\
B=\left[\begin{array}{c}
7.604 e+08 \\
-1.298 e+09 \\
8.822 e+08
\end{array}\right] \\
C=\left[\begin{array}{lll}
1 & 0 & 0 \\
0 & 1 & 0 \\
0 & 0 & 1
\end{array}\right]
\end{gathered}
$$

where the first, second and third rows of $\mathrm{C}$ can be taken according to the outputs drive shaft torsion, engine speed and wheel speed.

$G=B$

where $\mathrm{w}$ and $\mathrm{v}$ are state disturbances and measurement disturbances respectively. The estimated error covariance is found by solving the Riccati equation which is given by,

$$
P_{f}=\left[\begin{array}{ccc}
0.7486 & -1.3889 & 0.5609 \\
-1.3889 & 2.6328 & -0.8858 \\
0.5609 & -0.8858 & -0.8492
\end{array}\right]
$$

The measurement noise vectors $\mathrm{w}$ and $\mathrm{v}$ are generated using random signals. The covariance matrices $\mathrm{W}$ and $\mathrm{V}$ corresponding to $\mathrm{w}$ and $\mathrm{v}$ are given by,

$$
W=10^{4}
$$


$V=0.1$

The vehicle specifications used for the design of Kalman filter are given in Table 1.

\begin{tabular}{|l|l|}
\hline Mass of vehicle $(\mathrm{kg})$ & $m=1200$ \\
\hline Torsional stiffness of drive shaft $(\mathrm{Nm} / \mathrm{rad})$ & $k=200000$ \\
\hline Torsional damping of drive shaft $(\mathrm{Nm} /(\mathrm{rad} / \mathrm{s}))$ & $c=0.01$ \\
\hline Mass Moment of inertia of engine $\left(\mathrm{kgm}^{2}\right)$ & $J_{m}=0.16$ \\
\hline Mass Moment of inertia of transmission $\left(\mathrm{kgm}^{2}\right)$ & $J_{t}=0.034$ \\
\hline Mass Moment of inertia of final drive $\left(\mathrm{kgm}^{2}\right)$ & $J_{f}=0.034$ \\
\hline Mass Moment of inertia of wheel $\left(\mathrm{kgm}^{2}\right)$ & $J_{w}=0.8$ \\
\hline Final drive ratio & $i_{f}=3.1$ \\
\hline Radius of the Tyre $(\mathrm{m})$ & $r_{w}=0.4$ \\
\hline Viscous friction component of transmission $(\mathrm{Nms})$ & $b_{t}=0.04$ \\
\hline Viscous friction component of final drive $(\mathrm{Nms})$ & $b_{f}=0.04$ \\
\hline Viscous friction component of wheel $(\mathrm{Nms})$ & $b_{w}=0.06$ \\
\hline
\end{tabular}

Table 1: Vehicle driveline specifications.

The Kalman gain is found using these specifications which is given by,

$K_{f}=\left[\begin{array}{c}-173.7408 \\ 213.2491 \\ -359.8127\end{array}\right]$

The PID controller is designed to minimize the torque estimate by Kalman filter. The transfer function of the plant is found using the proposed state space model, which is given by,

$H(s)=\left(-1.358 e+07 s^{2}+2.114 e+07 s+1.397 e+07\right) /\left(s^{3}+1.141 s^{2}+10.09 s-5.823\right)$

\section{CONCLUSION AND RESULTS}

A model of manual transmission with engine, clutch, gear box, drive-shaft and vehicle body is constructed in simulink. Specifications for engine and all other components are given manually. Using this model different gears are engaged with corresponding clutch and throttle inputs. Then engine speed, fuel consumption, engine power, clutch speed, wheel speed etc are obtained from the model.

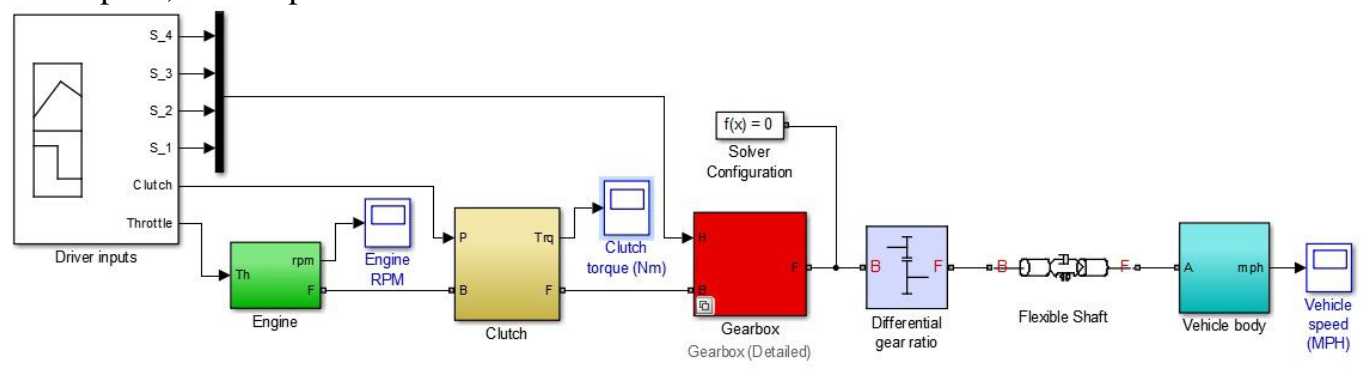

Figure 6: Model of Manual Transmission in Simulink.

The various inputs given by the driver such as gear engage and disengage, clutch engage and disengage, throttle action are given in Fig 7. 


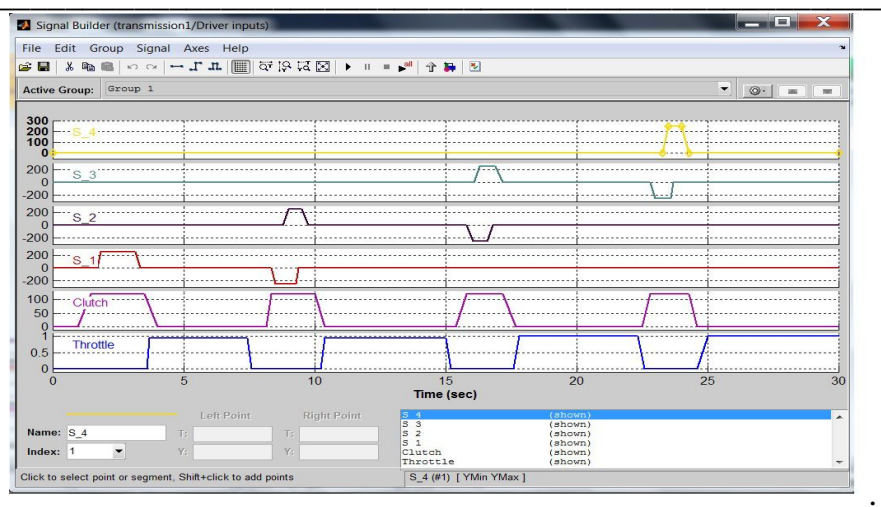

Figure 7: Inputs given by driver.

Using fuel consumption and measured engine speed as input and output, one system model is formulated in matlab. First the values of input-output variables are imported to matlab workspace and represented as iddata objects. Using the measured input and output, the parameters and states of the system are estimated. The obtained model is validated by comparing measured and simulated engine rpm values. After simulation in MATLAB, the strategy is implemented in LPC1768 using the development platform. The LPC1768 is an ARM Cortex-M3 based microcontroller for embedded applications featuring a high level of integration and low power consumption. The strategy should be tested in real vehicle environment. Such an environment is simulated using a LABCAR connected with ECU and PC. The LPC board is connected with ECU using CAN bus. First the LABCAR is connected with physical ECU using ecu harness. The LABCAR is connected with real time PC for viewing its response to the software, which is flashed in the ECU. After making all the connections power on the LABCAR. The program code needs readings from four sensors in the LABCAR. Therefore before powering it up, the working status of engine torque sensor, gear position sensor, engine speed sensor and wheel speed sensor are ensured and calibrated properly. The software for the complete operation of the vehicle is flashed into the ECU. The vehicle runs from start condition and gear change occurs according to the speed variation. The ECU is configured such that the sensors in LABCAR measure torque, speed values, store them in ECU ROM during the complete test and display in INCA if requested. After powering up the system, the vehicle (LABCAR) is allowed to run for 440 seconds. Gears are automatically engaged as per the speed variation. Keeping the clutch signal at zero level, torque, speed and other values are measured from sensors. For all $\backslash$ measurements, $\mathrm{x}$ axis represents time in seconds.

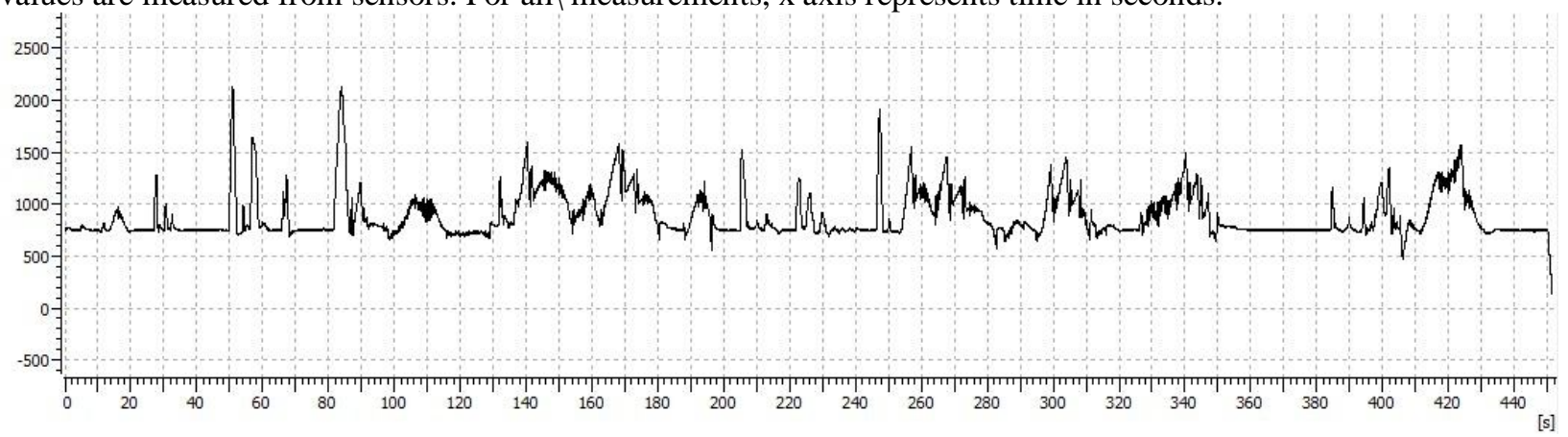

Figure 8: Engine speed. 


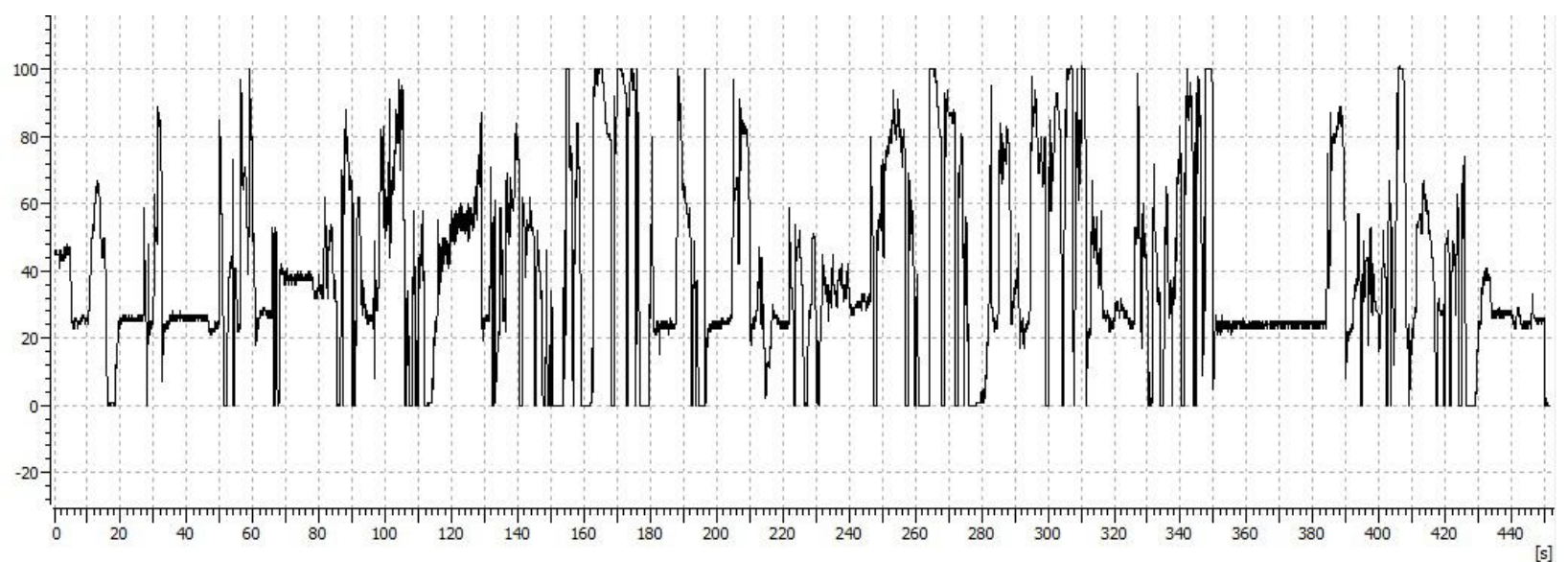

Figure 9: Vehicle speed

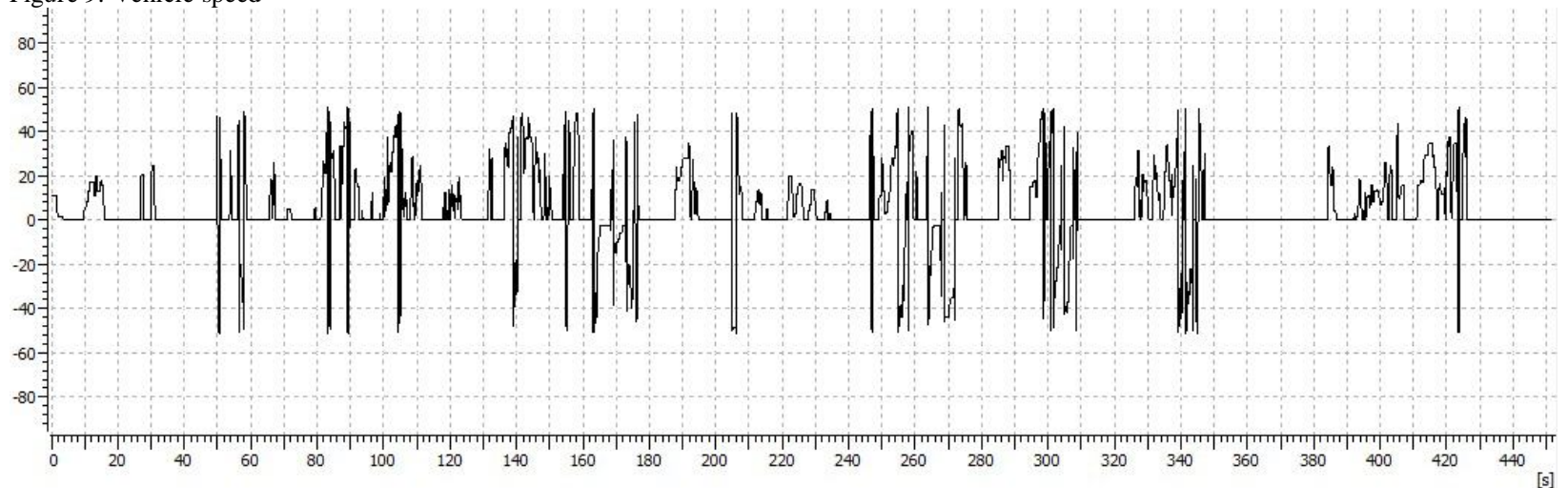

Figure 10: Accelerator pedal position.

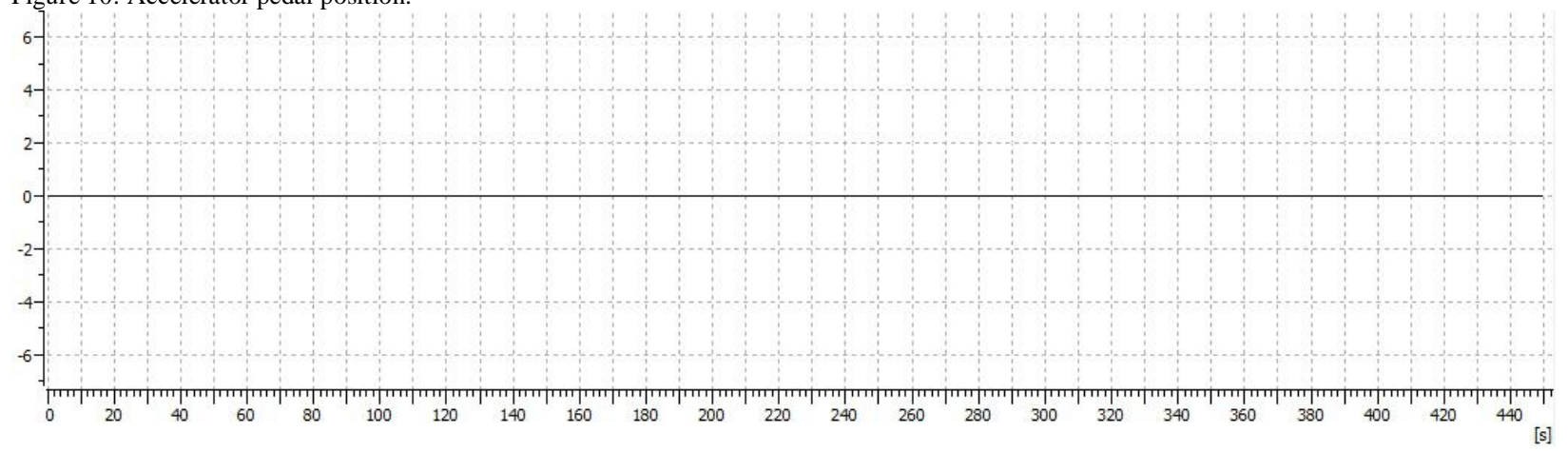

Figure 11: Clutch signal.

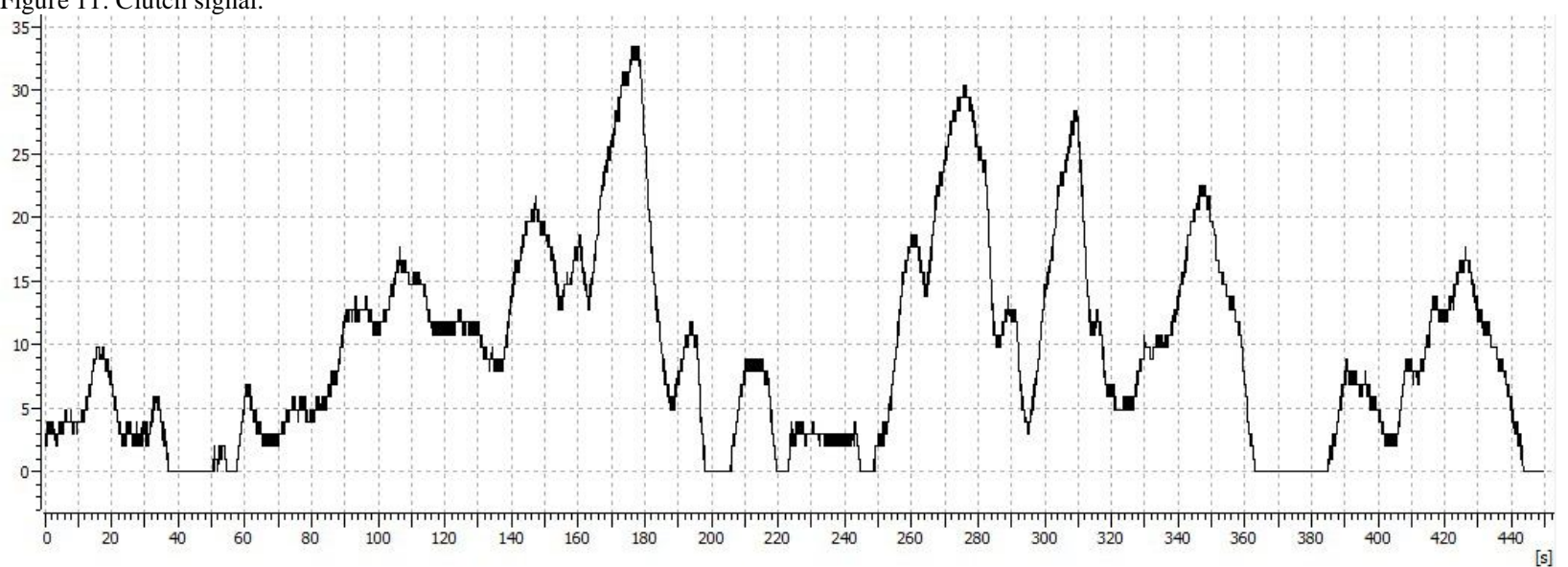


Design and Implementation of an Engine Control Strategy During Gear Shifting Process of Automated Manual Transmission (AMT) for Improvement of Shift Quality and Fuel Efficiency

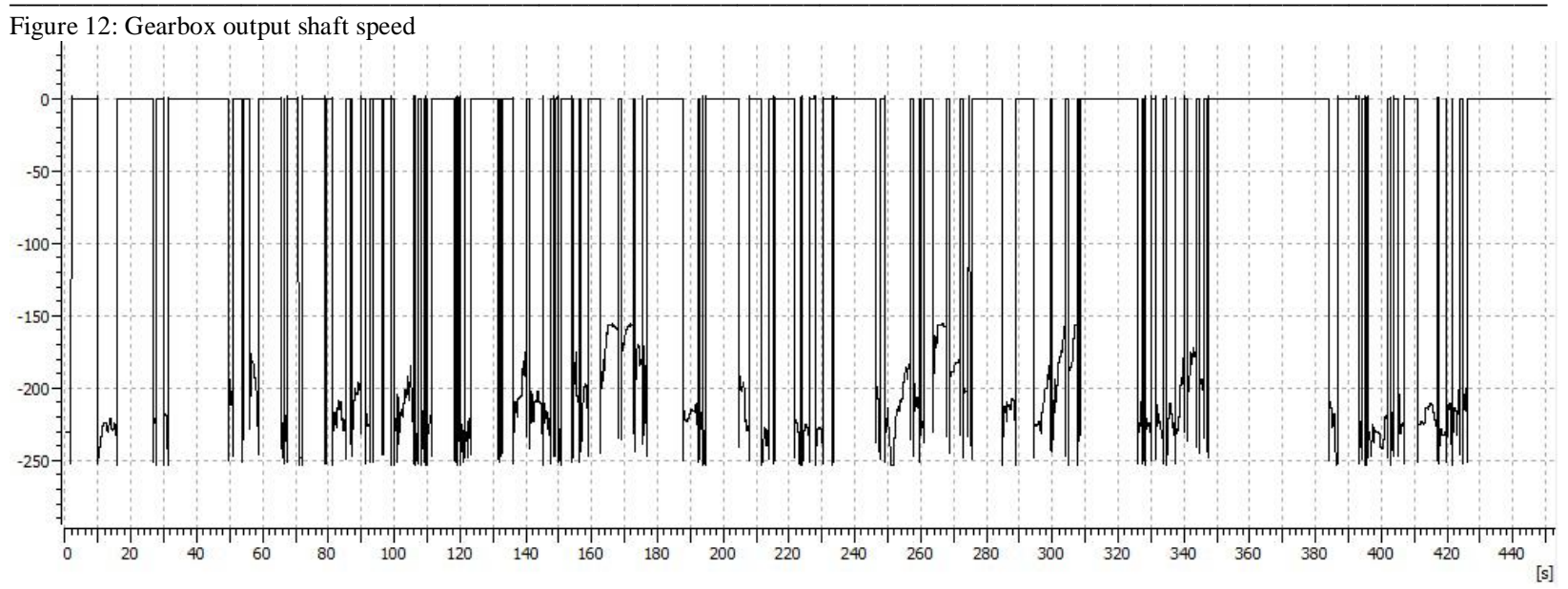

Figure 13: Driver demand torque.
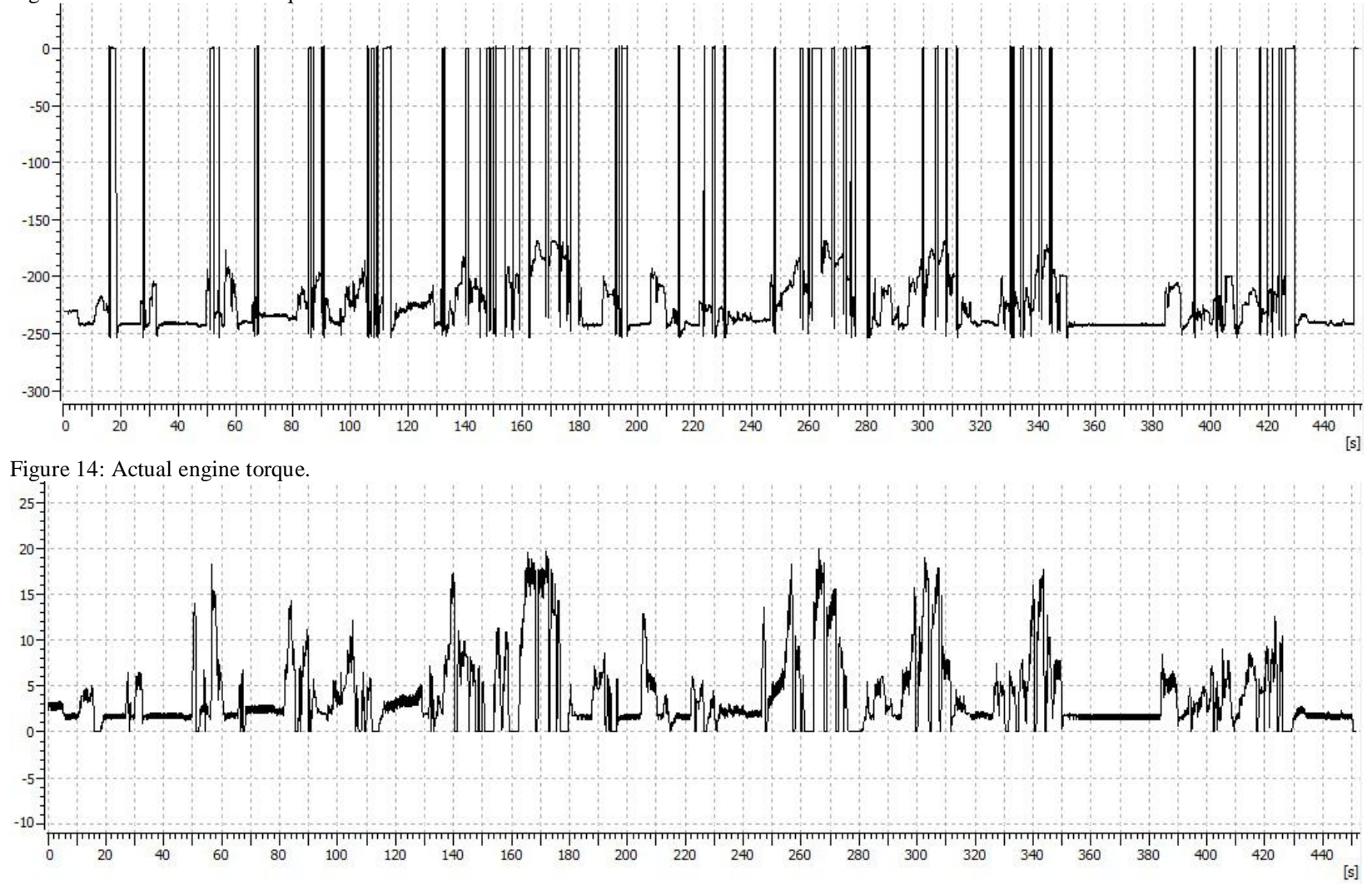

Figure 15: Fuel rate. 


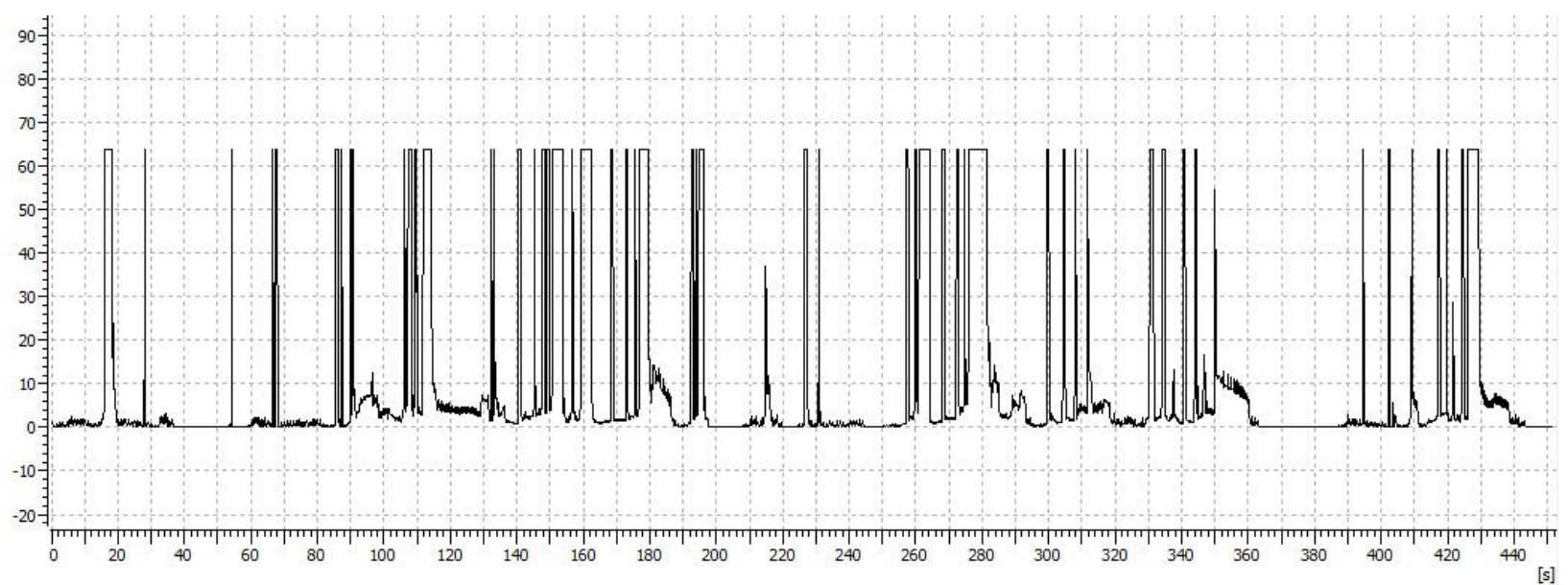

Figure 16: Instantaneous fuel economy.

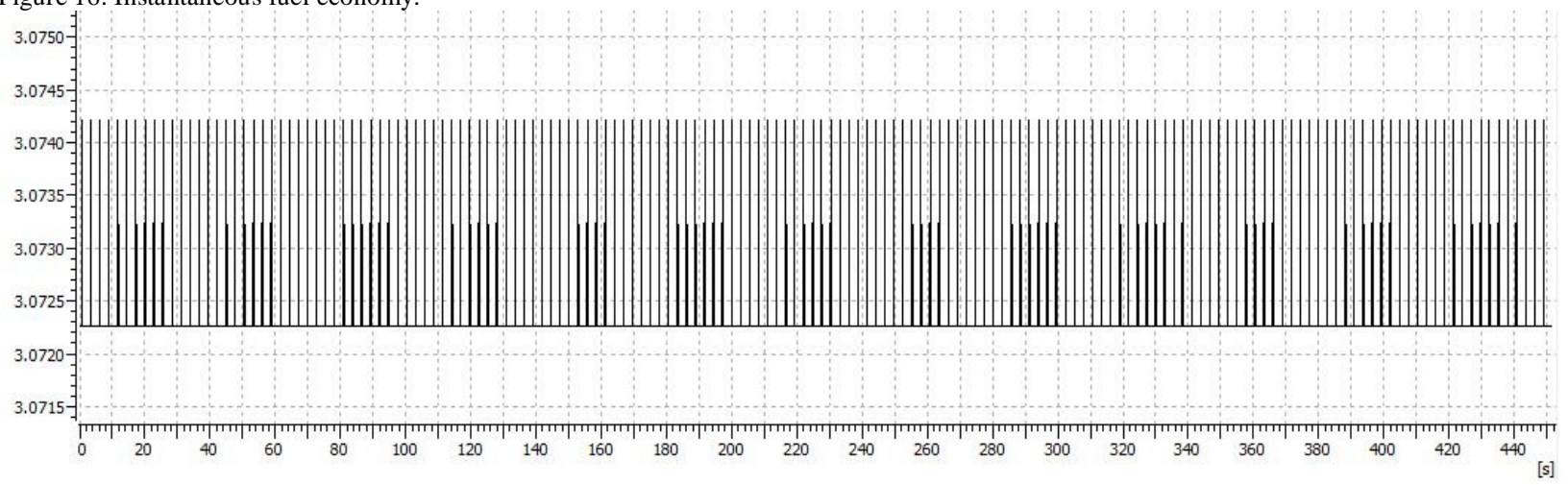

Figure 17:Average fuel economy.

\section{- Fuel Efficiency}

Fuel efficiency is the work output of an engine in terms of vehicle travel as a function of the energy content of the fuel expended in the operation of the vehicle. The fuel economy of a car can be enhanced by improving the fuel efficiency. To calculate the fuel efficiency, first the actual engine torque (Nm), engine speed (rpm) and fuel consumption rate $(\mathrm{gm} / \mathrm{s})$ are loaded from the graphics window to the trace window in INCA. For each variable sufficient number of samples are taken from the trace window. The engine power $(\mathrm{kW})$ is calculated from engine torque and engine rpm. Later efficiency is calculated from fuel consumption rate and engine power.

\section{REFERENCES}

[1] Z. Zhong, G. Kong, Z.Yu, X.Xin and X. Chen, "Shifting Control of an Automated Mechanical Transmission without using the Clutch", Int. J Automotive Tech, Vol.13 No 3 ,pp. 487-496, February, 2012.

[2] Magnus Pettersson and Lars Nielsen, "Gear Shifting by Engine Control", IEEE Transactions on Control Systems Technology, Vol. 8, No. 3, May 2000.

[3] Zaimin Zhong, Qiang Lv and Guoling Kong, "Engine Speed Control For The Automatic Manual Transmission During Shift Process", 2nd International Conference on Consumer Electronics, Communications and Networks (CECNet), 2012.

[4] Xiaofeng Yin, Dianlun Xue and Yun Cai, "Application of Time-Optimal Strategy and Fuzzy Logic to the Engine Speed Control during the Gear-Shifting Process of AMT", 4th International Conference on Fuzzy Systems and Knowledge Discovery (FSKD), Vol. 4, 2007.

[5] Magnus Pettersson, "Drive-line Modeling and Control", Linkoping Studies in Science and Technology, Dissertation No. 484, 1997.

[6] Uwe Kiencke and Lars Nielsen, "Automotive Control Systems For Engine, Driveline, and Vehicle", 2nd Edition, Springer Berlin Heidelberg New York, 2005.

[7] Luigi Glielmo, Luigi Iannelli, Vladimiro Vacca, and Francesco Vasca, "Gearshift Control for Automated Manual Transmissions", IEEE/ASME Transactions On Mechatronics, Vol. 11, No. 1, February 2006. 
Design and Implementation of an Engine Control Strategy During Gear Shifting Process of Automated Manual Transmission (AMT) for Improvement of Shift Quality and Fuel Efficiency

[8] Luigi Glielmo, Luigi Iannelli, Vladimiro Vacca, and Francesco Vasca, "Speed Control for Automated Manual Transmission with Dry Clutch", 43rd IEEE Conference on Decision and Control, Atlantis, Paradise Island, Bahamas, December 2004.

[9] Ngo V., Hofman T., Steinbuch M. and Serrarens A., "Optimal Control of the Gearshift Command for Hybrid Electric Vehicles", IEEE Transactions on Vehicular Technology, Vol. 61, No. 8, pp. 3531-3543, 2012.

[10] Van Reeven V., Hofman T., Huisman R. and Steinbuch M, "Extending Energy Management in Hybrid Electric Vehicles with explicit control of gear shifting and start-stop", IEEE Conference Publications on American Control Conference (ACC), pp. 521-526, 2012.

[11] Shusen Lin, Siqing Chang and Bo Li, "Gearshift System Design for Automated Manual Transmission based on an Electromagnetic Actuator", International Conference on Electrical and Control Engineering (ICECE), pp. 2250-2253, 2011.

[12] Dimitri P. Bertsekas, "Dynamic Programming and Optimal Control", 3rd Edition, Athena Scientific, Belmont, Massachusctts, 2005.

[13] Gene F. Franklin, J. David Powell and Michael L. Workman, "Digital Control of Dynamic Systems", 3rd Edition, Addison Wesley, 1997.

[14] Hyeoun-Dong Lee, Seung-Ki Sul, Han-Sang Cho and Jang-Moo Lee, "Advanced Gear Shifting and Clutching Strategy for Parallel Hybrid Vehicle with Automated Manual Transmission", IEEE Conference on Industry Applications, Vol. 3, pp. 1709-1713, 1998.

[15] Harald Naunheimer, Bernd Bertsche, Joyachim Ryborz and Wolfgang Novak, "Automotive Transmissions, Fundamentals, Selection, Design and Application.", 2nd Edition, Springer-Verlag Berlin Heidelberg 2011.

[16] Mathworks website. [Online]Available: "http://www.mathworks.in/products/sysid/"

[17] Mathworks website. [Online]Available: "http://www.mathworks.in/help/control/ug/kalman-filtering.html"

[18] "LPC1769/68/67/66/65/64/63 32-bit ARM Cortex-M3 microcontroller" Rev. 9.4 - 4 April 2014, Product data sheet.

[19] "UM10360, LPC176x/5x User manual" Rev. 3.1, 2 April 2014.

[20] "Automated ECU-software tests using LABCAR AUTOMATION V2.0 at Robert Bosch GmbH Chassis Systems Simulations" TU Berlin, March 2005, Peter Schubert, CS-SM/ESI3, Robert Bosch GmbH.

[21] "LABCAR-VDYM V5.0 - the bridge between Vehicle Component and ECU Development" Dr.-Ing. Gerd Wittler, SIMPACK User Meeting 2004.

[22] "LPCXpresso Base Board User’s Guide" Embedded Artists AB Södra Promenaden 51 SE-211 38 Malmö Sweden. 